\title{
A report of nontraumatic cortical subarachnoid hemorrhage and subsequent management
}

\section{David Qiyuan Mao ${ }^{*, 1}$, Daniel Addess' \& Helen Valsamis²}

\begin{abstract}
Aim: Report a case of cortical subarachnoid hemorrhage (cSAH) and discuss its management. Patient \& methods: A 66-year-old woman presents with acute onset left arm numbness and weakness. Initial head CT shows small hyperdensity in sulci typical for CSAH. Extensive workup with MRI, lumbar puncture and blood tests is performed. No signs of infection, vascular malformations, thrombosis or cancer are found. At outpatient follow-up, she is diagnosed with CSAH secondary to amyloid angiopathy. She is treated with gabapentin. Results \& conclusion: Diagnosis of $\mathrm{CSAH}$ is challenging given its subtle findings, and management is empiric as there are only a few case series in literature.
\end{abstract}

First draft submitted: 10 July 2016; Accepted for publication: 29 September 2016;

Published online: 21 October 2016

\section{Background}

Cortical subarachnoid hemorrhage (cSAH) is a rare form of subarachnoid hemorrhage characterized by unique imaging findings. Diagnosis is challenging given its subtle findings, and management is empiric as there are only a few case series in literature [1-6]. Incidence of this disease is not known, but case series estimate that cSAH represent $7.5-19 \%$ of all subarachnoid hemorrhage. Given that incidence of subarachnoid hemorrhage is $10-15$ per 100,000 person years, a rough estimate places incident of cSAH on order of 0.7-2.8 per 100,000 person years [7].

Initially, cSAH can present as a small, cortical hyperdensities found on CT head. Due to its size, it can be difficult to be seen and often requires MRI to further characterize the finding. Fluidattenuated inversion recovery and diffusion-weighted imaging sequences are more sensitive, and can show increased signal along one to a few sulci without spread to fissures, cisterns or ventricles [4]. Gradient echo (GRE) T2-weighted imaging can show hemoglobin degradation products, which are supportive of the presence of surface microbleeds [1]. GRE can also show hemosiderosis which occur outside time frame of acute bleed [2].

These imaging findings can be difficult to distinguish between other etiologies such as metastasis, cerebral venous thrombosis and vascular malformations. Additional testing is required to evaluate for alternative diagnoses [4]. In this case report, we discuss initial testing and long-term management.

\section{Case discussion}

Our patient was a 66-year-old right-handed woman with a history of hypertension, hyperlipidemia and breast cancer (currently in remission after left mastectomy years ago). The patient stated she woke up by a phone call at 06:30, and was at baseline. During the phone conversation, she developed

\section{KEYWORDS}

- cerebrovascular disease

- headache $\bullet$ imaging

- neuroimaging $\bullet$ seizures

- transient ischemic attack 
left arm numbness and weakness while holding her phone. Her symptoms were sudden onset and lasted approximately $10 \mathrm{~min}$ with spontaneous resolution. She did not have similar episodes in the past. Then, she had a second episode at 08:10, this time lasting approximately $40 \mathrm{~min}$. She delayed presenting to ED until later afternoon, $9 \mathrm{~h}$ after symptom onset. On review of systems, she also complained of a gradual onset, vertex headache that started after she noticed her numbness.

On neurologic examination, she was alert and oriented to person, place and time. Her speech was fluent, comprehension was intact and fund of knowledge was good. She had mild left facial asymmetry and left pronator drift that later resolved. She had no other cranial nerve or motor findings. Numbness resolved at time of initial evaluation, and there were no further sensory changes. The remainder of her examination was unremarkable. No early signs of acute stroke was found on head CT, but there was small hyperdensity in right parietal sulci. Tissue plasminogen activator (TPA) alteplase was not given.

The patient was admitted for further workup. MRI was negative for areas of restricted diffusion, but there was abnormal signal within the sulci of right parietal postcentral sulcus. This lesion was hyperintense on fluid-attenuated inversion recovery (Figure 1) and isointense on T1 (Figure 2). GRE shows positive signal in area of the lesion, but otherwise no additional microbleeds were found (Figure 3). Magnetic resonance angiogram of head and neck showed mild narrowing of the posterior circulation vessels, but otherwise there was no significant stenosis or aneurysm.

The differential diagnosis for this finding included subarachnoid hemorrhage, venous thrombosis, meningitis and carcinomatosis. Appropriate workup for these etiologies was pursued. MRI with contrast showed the lesion was enhancing, but no other enhancing lesions were found. Magnetic resonance venography was also performed, and was negative for venous thrombosis. She was further evaluated with cerebrospinal fluid study, which demonstrated: RBC 1, WBC 1, glucose 90 and protein 22. Cytology was negative for atypical cells. Infectious workup included testing for herpes simplex virus, West Nile, cytomegalovirus and varicella zoster virus, which were negative. Bacterial and fungal cultures were negative. After 1 week hospital stay, there were no further episodes of numbness or weakness, and the patient was discharged.

Patient was seen 2 weeks later at outpatient follow-up. Given her age, clinical picture and imaging finding or cortical sulci hemorrhage, she fulfilled Boston criteria for possible cerebral amyloid angiopathy (CAA) [8]. She was advised that she may need long-term follow-up for her increased risk of vascular complications and intracranial hemorrhage. In addition, she was started on oxcarbazepine for possible seizures as cause of her symptoms. It was later discontinued due to poor compliance. Subsequently, she was started on gabapentin, and had no
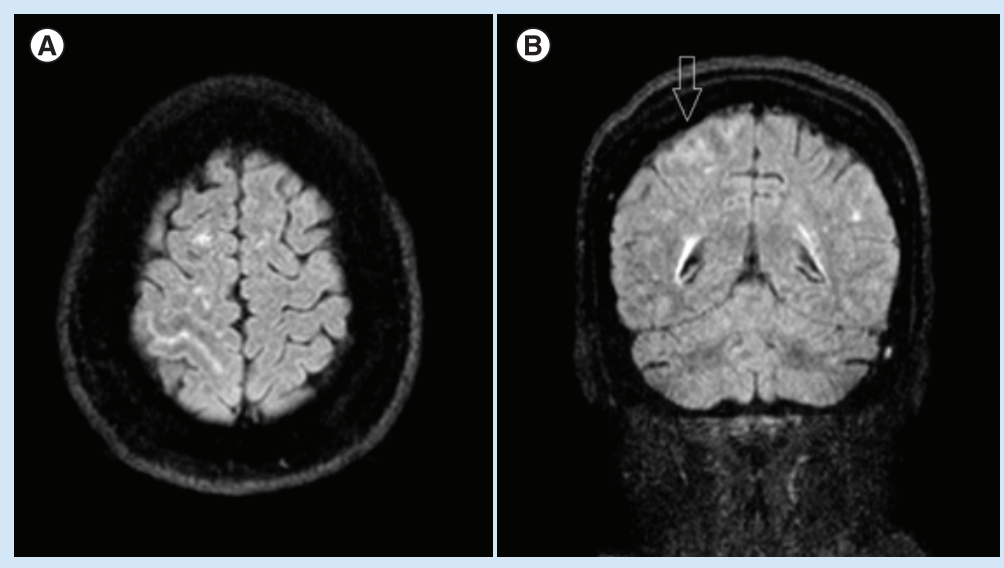

Figure 1. Fluid-attenuated inversion recovery imaging findings. (A) Axial FLAIR recovery shows linear hyperintensity in right parietal sulcus. (B) Arrow demonstrates finding on coronal FLAIR. FLAIR: Fluid-attenuated inversion recovery. 
further headache or focal neurologic deficits. At 6 months follow-up, she continued to report resolution of symptoms.

\section{Discussion}

A wide range of pathologies can cause cSAH, and the common ones found in case series include posterior reversible encephalopathy syndrome, vasospasm and CAA. Etiology may also be different with age. Kumar et al. found that in patients younger 60 , the most common cause of cSAH was reversible cerebral vasoconstriction syndrome, while CAA was the most common etiology observed in patients older than 60. One study used Boston criteria for diagnosis of possible CAA [2]. To fulfill this criteria, the patient must be older than 55 years of age, and clinical picture and imaging must fit the diagnosis. Other etiologies may present with similar imaging findings, and they need to be ruled out. These include cerebral venous thrombosis, coagulopathies, leptomeningeal metastasis and vascular malformations [8].

Given that cSAH can be secondary to any of a variety of causes, its prognosis and management rests on treating the primary disease whenever possible [9]. Its initial workup should screen for potential life-threatening conditions. Unlike conventional subarachnoid hemorrhage, cSAH is not associated with saccular aneurysm. However, vessel imaging with magnetic resonance angiogram, magnetic resonance venography and CT angiogram is critical in screening for other vascular abnormalities [6]. Conventional angiogram should be prioritized in younger patients as they have higher risk of reversible cerebral vasoconstriction syndrome [3].

MRI of brain can further characterize the lesion, and identify characteristic signs of associated conditions such as CAA. Next, consider lumbar puncture, echocardiogram and appropriate cancer screening to rule out infectious and neoplastic causes. If workup is negative, then long-term follow-up is needed as these patients have higher risk of stroke and intracerebral hemorrhage [6]. Last, this case report and others have shown that antiepileptic medications can treat recurrent focal neurologic symptoms associated with this disease.

\section{Conclusion}

The imaging findings in this case were typical for $\mathrm{cSAH}$. The lesion was found within one

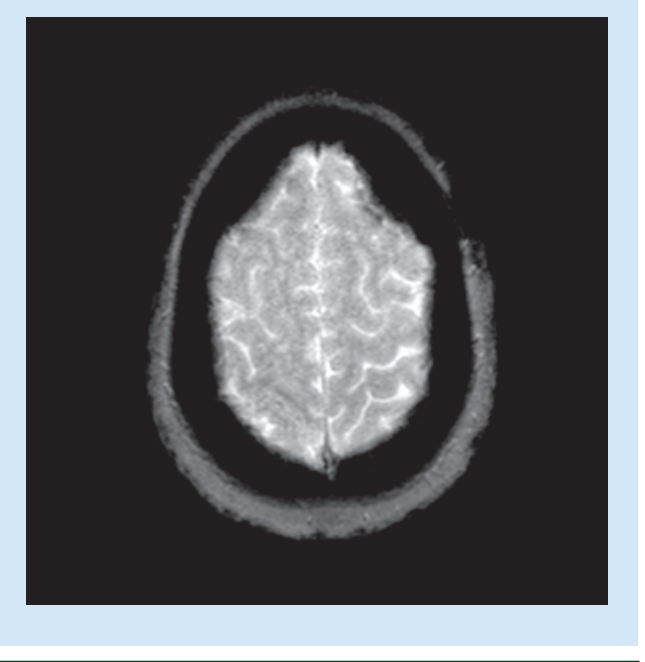

Figure 2. Finding is isointense on T1.

cortical sulcus and was small in size. Two common symptoms seen in the literature are severe headache and/or recurring transient focal neurologic symptoms (such as sensory/motor deficits and aphasia) [10]. The exact mechanism is unknown. Current hypotheses include spreading cortical depression, focal seizures or vasospasminduced transient ischemic attack [3]. In one case series, starting an antiepileptic drug was effective in preventing recurrent neurologic symptoms in four out of five patients [2]. This is only case report, that we know of, that shows gabapentin is an effective treatment after long-term follow-up.

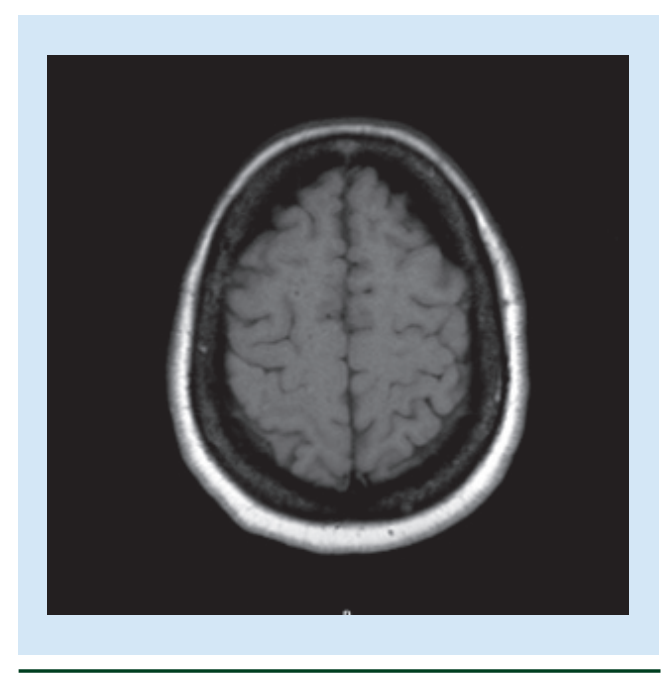

Figure 3. Gradient-recalled echo MRI demonstrates linear susceptibility in right parietal sulcus consistent with subarachnoid blood. 


\section{Future Perspective}

Current understanding of this condition are mainly derived from single-center restrospective reviews. These studies are limited by lack of standardized diagnostic testing and treatment regimen. In future, multi-center studies are needed to confirm single center observations on longer term prognosis, associated conditions, and optimal treatment. For patients with recurrent focal neurologic symptoms, anti-epileptic drug treatment will become more prevalent. As more patients are treated, information will become available regarding effectiveness of anti-epileptic drugs. Additional studies are needed regarding whether prophylactic treatment is beneficial and whether certain medication regimens are more effective than others.

\section{Author contributions}

DQ Mao drafted/revised this manuscript. D Addess drafted this manuscript. H Valsamis reviewed relevant studies and supervised clinical care.

Financial \& competing interests disclosure

The authors have no relevant affliations or financial involvement with any organization or entity with a financial interest in or financial conflict with the subject matter or materials discussed in the manuscript. This includes employment, consultancies, honoraria, stock ownership or options, expert testimony, grants or patents received or pending, or royalties.

No writing assistance was utilized in the production of this manuscript.

\section{Ethical conduct of research}

Standard protocol approvals, registrations and patient consents. Scope and nature of the report was discussed with the patient. No identifying information was used. Signed consent was obtained from the patient. The authors state that they have obtained appropriate institutional review board approval or have followed the principles outlined in the Declaration of Helsinki for all human or animal experimental investigations. In addition, for investigations involving human subjects, informed consent has been obtained from the participants involved.

\section{Open access}

This work is licensed under the Creative Commons Attribution-NonCommercial 4.0 Unported License. To view a copy of this license, visit http://creativecommons.org/ licenses/by-nc-nd/4.0/

\section{EXECUTIVE SUMMARY}

- Cortical subarachnoid hemorrhage (CSAH) can present with headache and recurrent focal neurologic symptoms.

- Key feature of CSAH is hemorrhage in one or a few sulci without extension into parenchyma or ventricles.

- $\mathrm{CSAH}$ is rare disease and management is tailored to the patient.

- Differential for imaging of cSAH includes infection, neoplasm and vascular disease.

- All patients with cSAH need MRI, vessel imaging and cerebrospinal fluid studies to rule out alternative disease processes.

- Older patients are more likely to have CSAH due to cerebral amyloid angiopathy. Younger patients are more likely to have CSAH due to vasospasm.

- Focal neurologic symptoms may be due to transient ischemic attack, seizure or spreading cortical depression.

- Case reports show antiepileptic drugs may be effective for preventing recurrent symptoms.

\section{References}

Papers of special note have been highlighted as:

- of interest; $\bullet$ of considerable interest

1 Beitzke M, Enzinger C, Wünsch G et al. Contribution of convexal subarachnoid hemorrhage to disease progression in cerebral amyloid angiopathy. Stroke 46(6), 1533-1540 (2015).

2 Raposo N, Viguier A, Cuvinciuc V et al. Cortical subarachnoid haemorrhage in the elderly: a recurrent event probably related to cerebral amyloid angiopathy. Eur. J. Neurol. 18, 597-603 (2011).
- Discusses treatment with antiepileptic medications.

3 Kumar S, Goddeau RP Jr, Selim MH et al. Atraumatic convexal subarachnoid hemorrhage: clinical presentation, imaging patterns, and etiologies. Neurology 74 , 893-899 (2010).

- A retrospective review of cases seen at major academic center in the USA and is largest cohort of patients in literature.

4 Cuvinciuc V, Viguier A, Calviere L et al. Isolated acute nontraumatic cortical subarachnoid hemorrhage. AJNR Am. J. Neuroradiol. 31, 1355-1362 (2010).

5 Refai D, Botros JA, Strom RG et al. Spontaneous isolated convexity subarachnoid hemorrhage: presentation, radiological findings, differential diagnosis, and clinical course. J. Neurosurg. 109, 1034-1041 (2008).

6 Geraldes R, Sousa PR, Fonseca AC et al. Nontraumatic convexity subarachnoid hemorrhage: different etiologies and outcomes. J. Stroke Cerebrovasc. Dis. 23 , e23-e30 (2014). 
7 Linn FH, Rinkel GJ, Algra A, van Gijn J. Incidence of subarachnoid hemorrhage: role of region, year, and rate of computed tomography: a meta-analysis. Stroke 27, 625-629 (1996).

8 Knudsen KA, Rosand J, Karluk D, Greenberg SM. Clinical diagnosis of cerebral amyloid angiopathy: validation of the Boston criteria. Neurology 56, 537-539 (2001).

9 Usmani N, Ahmad FU, Koch S. Convexity subarachnoid hemorrhage in ischemic stroke. J. Neurol. Sci. 348, 259-261 (2015).
10 Rico M, Benavente L, Para M et al. Headache as a crucial symptom in the etiology of convexal subarachnoid hemorrhage. Headache 54, 545-550 (2014). 